\title{
miRNA-335 and miRNA-182 affect the occurrence of tongue squamous cell carcinoma by targeting survivin
}

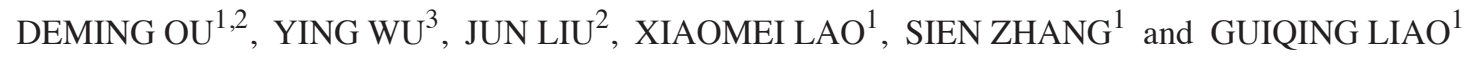 \\ ${ }^{1}$ Department of Oral and Maxillofacial Surgery, Guanghua School of Stomatology, \\ Guangdong Provincial Key Laboratory of Stomatology, Sun Yat-Sen University, Guangzhou, Guangdong 510055; \\ ${ }^{2}$ Department of Stomatology, Central Hospital of Panyu, Guangzhou, Guangdong 511400; ${ }^{3}$ Department of Stomatology, \\ Foshan Hospital of Chinese Traditional Medicine, Foshan, Guangdong 528000 P.R. China
}

Received February 10, 2015; Accepted March 15, 2016

DOI: $10.3892 / 01.2016 .4938$

\begin{abstract}
The aim of the present study was to characterize the roles of two microRNAs (miRs) that have been reported to be differentially expressed in tongue squamous cell carcinoma (TSCC), miR-335 and miR-182. In total, 20 tumor tissue samples and 20 corresponding adjacent non-cancerous samples were collected from patients with TSCC to measure the expression of miR-335 and miR-182 and the potential shared target of these miRs, survivin, using reverse transcription-quantitative polymerase chain reaction and western blotting. In the TSCC tissue samples, significantly decreased expression of the two miRs and increased expression of survivin were detected compared with adjacent non-cancerous controls. Subsequently, it was confirmed that survivin was the target gene of miR-335 and miR-182 using a luciferase assay in TSCC cells. In order to examine the function of miR-335 and miR-182 in the development of TSCC, TSCC cells were transiently transfected with the mimics of the two miRs, and it was confirmed that the introduction of miR-335 and miR-182 to cells suppressed the expression of survivin and markedly inhibited the proliferation of the TSCC cells. Furthermore, miR-335 and miR-182 were found to induce cell cycle arrest by suppressing the expression of survivin. The present study revealed a negative regulatory role of miR-335 and miR-182 in the proliferation of TSCC cells by targeting survivin, and miR-335 and miR-182 may be novel therapeutic targets for the treatment of TSCC.
\end{abstract}

\section{Introduction}

Tongue squamous cell carcinoma (TSCC) is the leading type of cancer of the oral cavity and is characterized by a high rate

Correspondence to: Dr Guiqing Liao, Department of Oral and Maxillofacial Surgery, Guanghua School of Stomatology, Guangdong Provincial Key Laboratory of Stomatology, Sun Yat-Sen University, 56 Lingyuanxi Road, Guangzhou, Guangdong 510055, P.R. China E-mail: stomatology123@163.com

Key words: miR-335, miR-182, tongue squamous cell carcinoma, survivin of tumor cell proliferation and metastasis (1). Approximately one-half of patients are already in advanced stages of disease at the time of diagnosis $(2,3)$. Elucidation of the molecular mechanism underlying the development and progression of TSCC may aid improved understanding of the pathogenesis of TSCC and improve the diagnosis, treatment and prevention of the disease.

MicroRNAs (miRNAs) are a class of endogenously expressed 22-nucleotide non-coding RNAs that are able to regulate gene expression by binding the 3 '-untranslated regions (3'-UTRs) of their target messenger RNAs, resulting in translational repression or mRNA degradation (4). It is hypothesized that approximately one-third of human genes can be regulated by miRNAs (4). Due to the various reported roles of miRNAs in the regulation of gene expression, miRNAs are considered to play key roles in numerous biological processes, including cell growth, apoptosis, metabolism and transformation (5-7). It has been reported that chromosome 7q32 is largely involved in the pathogenesis of malignancy (8). Previously, 8 miRNAs, consisting of miR-593, miR-129-1, miR-335, miR-182, miR-96, miR-183, miR-29a and miR-29b-1, were identified on this genomic locus, with certain miRNAs being recognized either as oncogenes or tumor suppressor genes (9-11). In the present study, 20 tumor and adjacent non-cancerous control tissue samples were collected from 20 patients with histologically confirmed TSCC. Confirmatory reverse transcription-quantitative polymerase chain reaction (RT-qPCR) showed that miR-335 and miR-182 were significantly downregulated in the tumor tissue samples compared with the controls. Notably, survivin was identified as a potential shared target of miR-335 and miR-182 by using online prediction tools (mirdb.org/miRDB/) and in silico analysis. Survivin is a member of the inhibitor of apoptosis protein (IAP) family and is well-known for its exclusive expression in fetal tissue and a wide spectrum of human cancers, but not in non-proliferating adult tissue (12). Survivin has also been reported to be a regulator of cellular proliferation and metastasis in cancer (13), and an increasing number of studies indicated that survivin was involved in various types of cancer (14-16), particularly TSCC $(2,3)$.

Based on the aforementioned evidence and the studies reporting that significant underexpression of miR-335 or 
miR-182 was noted in various types of cancers, including glioma (17), breast cancer (18) and gastric cancer (19), the present study hypothesized that miR-335 and miR-182 may function as regulators of the behavior of TSCC cells by modulating survivin. To test this hypothesis, the effect of miR-335 and miR-182 on the proliferation and invasion of TSCC cells was evaluated. The expression patterns of miR-335, miR-182 and survivin were also examined in the cancer tissues and compared with adjacent non-cancerous tissues.

\section{Materials and methods}

Tissue samples. Paired primary TSCC samples and adjacent histological normal tissues were collected from 20 TSCC patients, consisting of 14 male and 6 female patients (age, $62.36 \pm 5.74$ years), at the Department of Oral and Maxillofacial Surgery, Guanghua School of Stomatology, Sun Yat-sen University (Guangzhou, China), between September 2006 and December 2011. Tumor tissues and adjacent normal tissues that were at least $1.5 \mathrm{~cm}$ distal to the tumor margins were snap-frozen in liquid nitrogen and then stored at $-80^{\circ} \mathrm{C}$ until use. All samples were evaluated by two pathologists according to the World Health Organization guidelines and pathological tumor-node-metastasis Union for International Cancer Control pathological staging criteria (20), and the samples were examined for the expression level of survivin, miR-335 and miR-182. Patients that received chemotherapy or radiotherapy prior to surgery were excluded from the present study. Informed consent was obtained from all patients, and the study was approved by the Human Research Ethics Committee of Sun Yat-Sen University.

Cell culture. The human TSCC SCC-15 cell line was purchased from American Type Culture Collection (ATCC, Manassas, VA, USA). The SCC-15 cells were cultured in Dulbecco's modified Eagle's medium (DMEM; Gibco; Thermo Fisher Scientific, Inc., Waltham, MA, USA) supplemented with $10 \%$ heat-inactivated fetal bovine serum (FBS; Gibco; Thermo Fisher Scientific, Inc.), $100 \mathrm{U} / \mathrm{ml}$ penicillin (Gibco; Thermo Fisher Scientific, Inc.) and $100 \mu \mathrm{g} / \mathrm{ml}$ streptomycin (Gibco; Thermo Fisher Scientific, Inc.) at $37^{\circ} \mathrm{C}$ in a $5 \% \mathrm{CO}_{2}$ atmosphere.

Methyl thiazolyl tetrazolium (MTT) assay. The SCC-15 cells were transfected with miR-335 mimics, miR-182 mimics or the negative control (scramble control sequence) and incubated for $48 \mathrm{~h}$ at room temperature. Subsequently, $30 \mu 10.5 \%$ MTT (Sigma-Aldrich, St. Louis, MO, USA) solution was added to each well and the wells were incubated for another $4 \mathrm{~h}$. The medium was then replaced with $150 \mu$ l dimethyl sulfoxide (DMSO; Sigma-Aldrich) in the microplate, and then agitated on a rotary platform for $10 \mathrm{~min}$. The survival rate was determined by measuring the optical density (OD) values of the mimic-transfected cells relative to the OD of the control at a 492-nm wavelength using a Jenway spectrophotometer 7300 (67 series VU Visible Scanning spectrophotometer; Bibby Scientific Ltd., Stone, Staffordshire, UK).

Total RNA isolation and RT-qPCR. Total RNA was obtained using TRIzol One-Step RNA Isolation kit (Invitrogen; Thermo
Fisher Scientific, Inc.). Complementary DNA specific for miR-335, miR-182, survivin and glyceraldehyde 3-phosphate dehydrogenase (GAPDH), which acted as an internal control, was synthesized from total RNA using gene-specific primers with the TaqMan MicroRNA assay, according to the manufacturer's protocol (Applied Biosystems; Thermo Fisher Scientific, Inc.). Relative quantification of target miRNA expression was measured using the $2^{-\Delta \Delta \mathrm{Cq}}$ method (21). Each sample was examined in triplicate and the raw data were expressed as the relative quantity of target miRNA, normalized with respect to GAPDH.

Luciferase reporter assay. The SCC-15 cells were cultured in DMEM supplemented with $10 \% \mathrm{FBS}$ at $37^{\circ} \mathrm{C}$ in an atmosphere of $5 \% \mathrm{CO}_{2}$ to $70-80 \%$ confluency. Using Lipofectamine 2000 (Invitrogen; Thermo Fisher Scientific, Inc.), the cells were then transfected with the WT-Sur-UTR DNA plasmid, which consisted of a firefly luciferase reporter vector containing the wild-type survivin 3'-UTR, or the mutant Sur-UTR DNA plasmids (Mut1 and Mut2), which consisted of a firefly luciferase reporter vector containing the mutant survivin 3'-UTR, (Fig. 1) and the control pRL-TK vector, which contained Renilla luciferase (Promega Corporation, Madison, WI, USA). The firefly and Renilla luciferase activities were measured consecutively using Dual Glo Luciferase assays (Promega Corporation) $48 \mathrm{~h}$ subsequent to transfection.

Western blotting. Lysates were resolved on $12 \%$ sodium dodecyl sulfate-polyacrylamide gels and the bands were transferred onto polyvinylidene fluoride membranes (EMD Millipore, Billerica, MA, USA). The membranes were blocked with $5 \%$ non-fat milk at room temperature for $1 \mathrm{~h}$ followed by incubation with a rabbit anti-human survivin antibody (catalog no., ab2050; Abcam, Cambridge, UK) under room temperature for $2 \mathrm{~h}$ and subsequently incubated for $2 \mathrm{~h}$ with horseradish peroxidase-conjugated anti-rabbit secondary antibody at room temperature for $1 \mathrm{~h}$. Chemical fluorescence was detected using an Enhanced Chemiluminescence Western Blotting Detection Reagent kit (GE Healthcare, Little Chalfont, Buckinghamshire, UK), according to the manufacturer's protocol. The target bands underwent densitometric analysis with Quantity One ${ }^{\circledR} 1-\mathrm{D}$ analysis software version 170-9600 (Bio-Rad Laboratories, Inc., Hercules, CA, USA) and were normalized against $\beta$-actin. A total of 3 independent experiments were performed.

Cell cycle analysis and apoptosis analysis. The SCC-15 cells were digested with trypsin-ethylenediaminetetraacetic acid solution (Beyotime Institute of Biotechnology, Haimen, China), washed with phosphate-buffered saline (PBS; Invitrogen; Thermo Fisher Scientific, Inc.), and fixed in $70 \%$ ethanol $48 \mathrm{~h}$ subsequent to transfection. Cell cycle analyses were conducted using propidium iodide staining. FACSCalibur flow cytometer (BD Biosciences, San Jose, CA, USA) was used to determine $\mathrm{G} 0 / \mathrm{G} 1, \mathrm{~S}$ and $\mathrm{G} 2 / \mathrm{M}$ fractions. For the apoptosis analysis, an Annexin V-FLUOS Staining kit purchased from Roche (Mannheim, Germany) was used $48 \mathrm{~h}$ subsequent to transfection, according to the manufacturer's protocol. The results were analyzed using CellQuest Pro software (BD Biosciences). 
A

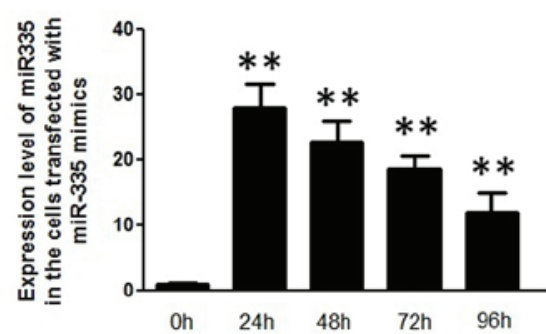

C

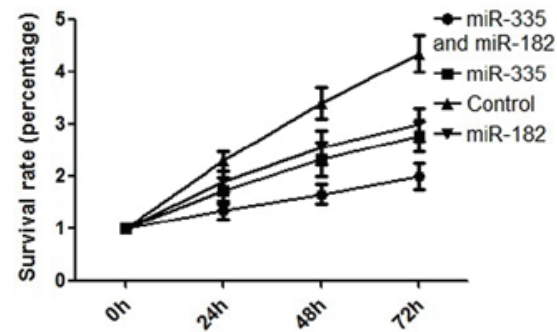

$\mathbf{E}$

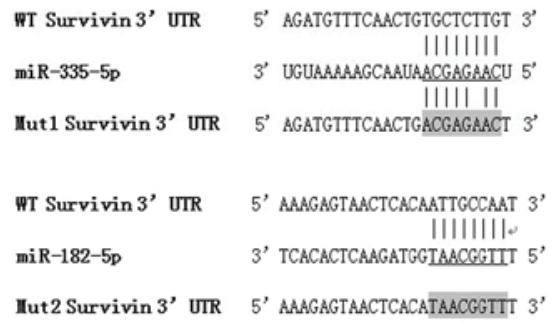

B

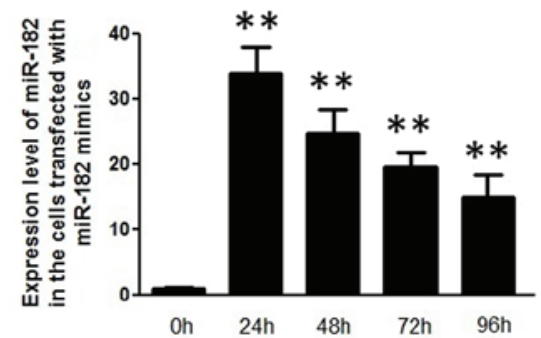

D

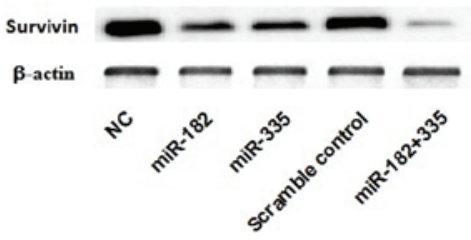

F

\begin{tabular}{|c|c|c|c|}
\hline \multirow[b]{2}{*}{ 驵 Survivin 3' UTR } & \\
\hline & Luciferase & Survivin 3'UTR & PolyA \\
\hline \multirow{3}{*}{ Iut1 Survivin 3' UIR } & \multicolumn{3}{|c|}{ ACGAGAAC } \\
\hline & Luciferase & Survivin 3'UTR & PolyA \\
\hline & \multicolumn{3}{|c|}{ TAACGGTT } \\
\hline Ilut2 Survivin 3' UTR & Luciferase & Survivin 3'UTR & PolyA \\
\hline
\end{tabular}
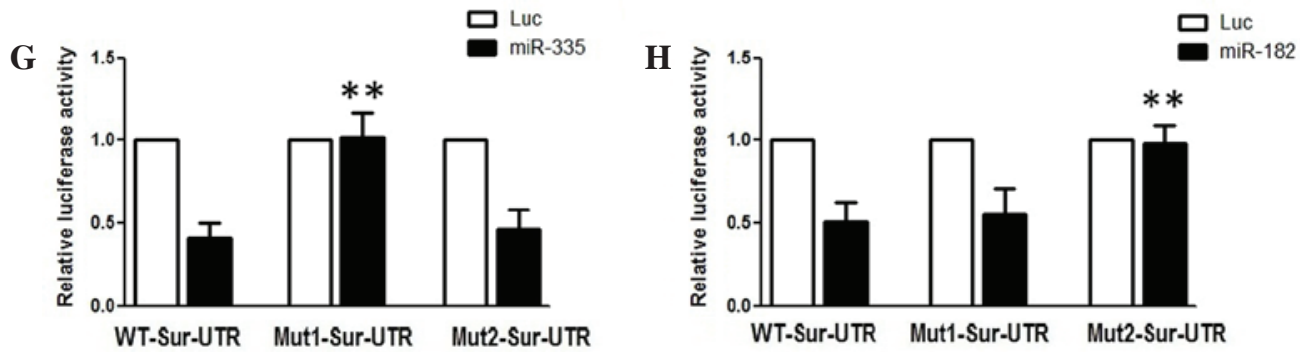

Figure 1. (A) Expression levels of miR-335 24, 48, 72 and $96 \mathrm{~h}$ subsequent to transfection with miR-335 mimics. (B) Expression levels of miR-182 24,48, 72 and 96 subsequent to transfection with miR-182 mimics. (C) Survival rate analysis of TSCC cells transfected with miR-335 and/or miR-182 mimics, as determined using a methyl thiazolyl tetrazolium assay 24,48 and $72 \mathrm{~h}$ subsequent to transfection. (D) Suppression of the protein expression levels of Sur by miR-335 and/or miR182 mimics in TSCC cells. (E) Schematic representation of miR-335 or miR-182 and the binding sequences of these miRs in the WT or Mut 3'-UTR of Sur mRNA. (F) Schematic representation of the Mut 3'-UTR of Sur mRNA. (G) Analysis of Luc activity in TSCC cells overexpressing miR-335 $48 \mathrm{~h}$ subsequent to cotransfection with the control Renilla Luc expression construct pRL-TK and firefly Luc reporter plasmids containing either WT or Mut 3'-UTR of Sur (H) Analysis of Luc activity in miR-182-overexpressing TSCC cells $48 \mathrm{~h}$ subsequent to cotransfection with the control Renilla Luc expression construct pRL-TK and firefly Luc reporter plasmids containing either WT or Mut 3'-UTR of Sur. ${ }^{* *} \mathrm{P}<0.01$ vs. control. miR, microRNA; TSCC, tongue squamous cell carcinoma; UTR, untranslated region; mRNA, messenger RNA; Mut, mutant; WT, wild-type; NC, negative control; Luc, luciferase; Sur, survivin.

Cell invasion assay. For the cell invasion assay, $8-\mu \mathrm{m}$ pore polycarbonate membrane filters (BD Biosciences) pre-coated with $50 \mu$ l Matrigel $(1.25 \mathrm{mg} / \mathrm{ml}$; BD Biosciences) were used. The cells transfected with each miR were collected and seeded at a density of $1 \times 10^{5}$ cells/well with $1 \%$ FBS-supplemented DMEM in the cell culture chambers, with the bottom chambers filled with $0.6 \mathrm{ml}$ medium. The chamber was incubated at $37^{\circ} \mathrm{C}$ for $48 \mathrm{~h}$ prior to the removal of the Matrigel. The invaded cells were fixed, stained and calculated. The invasiveness of cells was evaluated by the following equation: Invasion index $(\%)=$ invaded cell number / total cell number $\mathrm{x} 100$.

Statistical analysis. The data were expressed as the mean \pm standard deviation. The differences were assessed for significance using Student's $t$-test, $\chi^{2}$ test or one-way analysis of variance followed by Fisher's least significant difference test, as appropriate. The statistical analysis was conducted by SPSS 17.0 software (SPSS, Inc., Chicago, IL, USA). P $<0.05$ was considered to indicate a statistically significant difference.

\section{Results}

Overexpression of miR-335 and miR-182 suppresses the growth of TSCC cells. To evaluate the effect of miR-335 and miR-182 on the proliferation and invasion of TSCC cells, the SCC-15 cells were transfected with miR-335 and miR-182 mimics. As shown in Fig. 1A and B, the levels of miR-335 and miR-182 were increased $>30$-fold at $24 \mathrm{~h}$ subsequent to 
transfection compared with the control. The MTT assay results showed that exogenous overexpression of miR-335 and miR-182 individually and synergistically suppressed the proliferation of SCC-15 cells compared with the control (Fig. 1C). In addition, the migratory ability of the cells with or without overexpression of miR-335 and miR-182 was examined, and the introduction of either miR-335 or miR-182 was not found to affect the migratory ability of the cells (data not shown). The results indicated that miR-335 and miR-182 each played inhibitory roles in the regulation of cell proliferation.

Exogenous overexpression of $m i R-335$ and miR-182 led to G1 phase cell cycle arrest in TSCC cells. To investigate the mechanism underlying the effect of miR-335 and miR-182 on cell proliferation, cell cycle distribution analysis was performed in TSCC cells using flow cytometry. The data revealed that overexpression of miR-335 and/or miR-182 arrested the growth of TSCC cells in the G1 phase, with a decrease in the proportion of cells in the G2/M and S phases (Fig. 2). The result showed that compared with the control group, the percentage of TSCC cells in the G1 phase was increased between 61.35 and $75.14 \%$ in cells transfected with miR-335 alone $(\mathrm{P}<0.01), 61.35$ and $72.11 \%$ in cells transfected with miR-182 alone $(\mathrm{P}<0.01)$ and 61.35 and $88.32 \%$ in cells transfected with miR-335 combined with miR-182 $(\mathrm{P}<0.01)$. The percentage of cells in the $\mathrm{S}$ phase decreased between 29.26 and $16.24 \%$ in cells transfected with miR-335 alone $(\mathrm{P}<0.01), 29.26$ and $19.50 \%$ in cells transfected with miR-182 alone $(\mathrm{P}<0.01)$ and 29.26 and $4.23 \%$ in cells transfected with miR-335 combined with miR-182 $(\mathrm{P}<0.01)$. The population of cells in the G2 phase decreased between 9.39 and $8.63 \%$ in cells transfected with miR-335 alone $(\mathrm{P}<0.01)$, between 9.39 and $8.39 \%$ in cells transfected with miR-182 alone $(\mathrm{P}<0.01)$ and between 9.39 and $7.45 \%$ in cells transfected with miR-335 and miR-182 (P<0.01)(Fig. 2A-D). These data demonstrated that overexpression of miR-335 and miR-182 suppressed the proliferation of TSCC cells by arresting cell growth in the G1 phase.

Survivin is a shared direct target of miR-335 and miR-182 in TSCC cells. The miRNA databases miRanda (22) and TargetScan (23) were searched for potential target genes of miR-335 and miR-182, in order to identify the mediators of the inhibitory effect of miR-335 and miR-182 on cell proliferation. By screening the candidate genes that were identified based on their functions combined with the information about the miRNA obtained from the DIANA lab-based microRNA pathway analysis tool (diana.imis.athena-innovation.gr/DianaTools/index.php), all predicted genes were categorized functionally to identify candidate genes that are functionally associated with the regulation of the cell cycle. Finally, the present study focused on survivin, a key cell cycle regulator and a putative shared target of miR-335 and miR-182 (Fig. 1E). Therefore, it was hypothesized that miR-335 and miR-182 may participate in the regulation of the cell cycle by targeting survivin. To test the hypothesis, the present study measured and compared the expression level of survivin in the TSCC cells transfected with miR-335 and miR-182 mimics using western blot analysis. The effects of these mimics on survivin knockdown were comparable with the survivin level in cells co-transfected with the two
miRs, which demonstrated an additional decrease (Fig. 1D). Furthermore, a luciferase assay was conducted to confirm that survivin is a direct shared target of miR-335 and miR-182. Wild-type or mutant survivin 3'-UTR was cloned downstream of a firefly luciferase gene (Fig. 1F), and the constructs were transfected into miR-335 or miR-182-overexpressing cells or the negative control. In addition, overexpression of miR-335 significantly decreased the relative luciferase activity of the wild-type and Mut1 survivin 3'-UTR, but had minimal effects on the relative luciferase activity of the Mut 2 survivin 3'-UTR (Fig. 1G). Overexpression of miR-182 suppressed the luciferase activity of wild-type and Mut2 (Fig. 1H), but the luciferase activity of Mut1 was almost unaltered, suggesting that miR-335 and miR-182 suppress the expression of survivin by binding directly to the corresponding 'seed sequence' in the 3'-UTR of survivin.

Examination and comparison of expression patterns of survivin and miR-335/miR-182 in cancerous tissue and adjacent normal tissue. The levels of miR-335, miR-182 and survivin were measured and compared in cancerous tissues $(n=20)$ and adjacent normal tissues $(n=20)$. As shown in Fig. 2E, it was found that the relative expression level of miR-335 and miR-182 in cancerous tissue was significantly downregulated to $\sim 20$ and $10 \%$, respectively, compared with normal tissues. The expression level of survivin mRNA was measured by RT-qPCR, and it was found that the mRNA level of survivin in the cancerous tissue was significantly increased compared with normal tissues (Fig. 2F). The survivin protein level was also measured using western blotting, and the relative density of survivin in the cancerous tissue was almost 5-fold higher compared with the normal control (Fig. 2G and H).

\section{Discussion}

RNA-based therapeutic strategies, such as siRNA and miRNA, depict a promising future for cancer treatment by regulating target genes that are involved in cellular activities, such as cell growth, apoptosis, metabolism and transformation (5-7). Therefore, investigation of miRNAs and the targets and roles of miRNAs in the regulation of cell behavior is crucial to improve the understanding and facilitate the clinical application of RNA-based therapeutic strategies.

At present, a panel of miRNAs have been reported as either tumor suppressors, as they are downregulated, or oncogenes, as they are upregulated, in multiple human cancers (24). miR-335 and miR-182 have been shown to be involved in the regulation of cancer cell proliferation and progression, but the roles of miR-335 and miR-182 in the development of cancer were inconsistent among various cancer types (25). miR-335 functions as a tumor suppressor in osteosarcoma by regulating survivin expression (25). However, Lu et al (26) reported that overexpression of miR-335 promotes cell proliferation and tumor growth of colorectal carcinoma cells. Furthermore, miR-182 was reported to exhibit anticancer activity in cervical cancer by inducing cell apoptosis via the inhibition of DNMT3a expression (27). By contrast, miR-182 promotes cell growth, migration and invasion of prostate cancer via the suppression of FOXO1 expression (28). As 
A

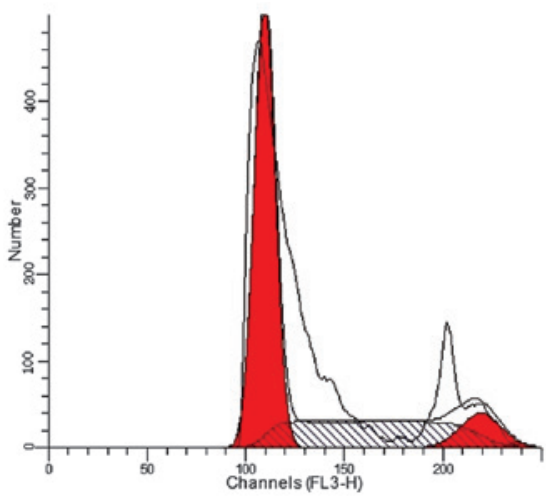

C

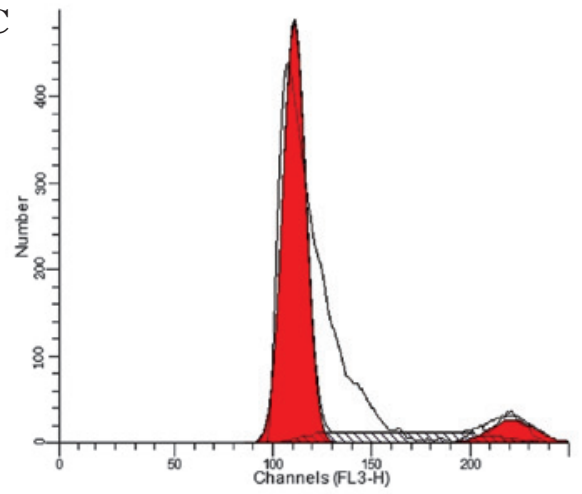

$\mathbf{E}$

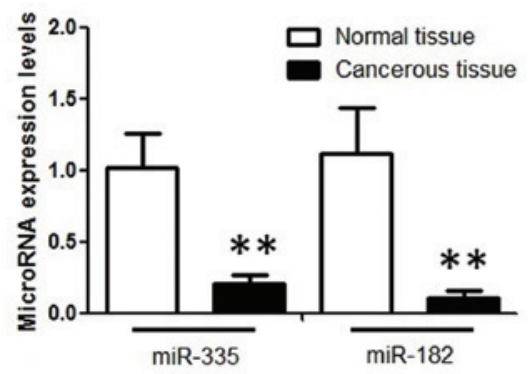

G

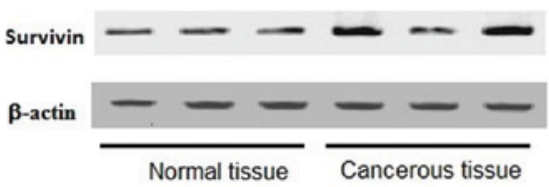

B

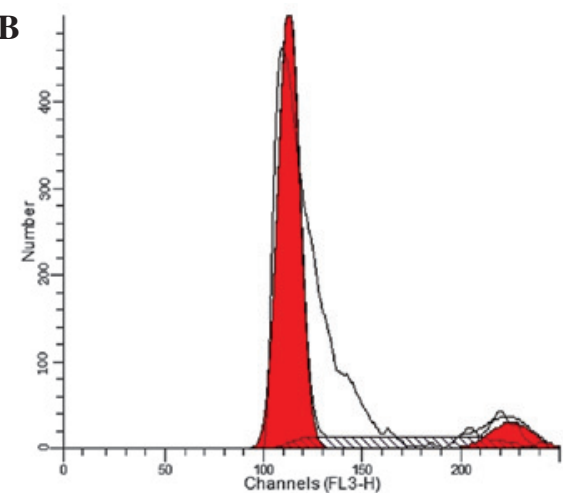

D

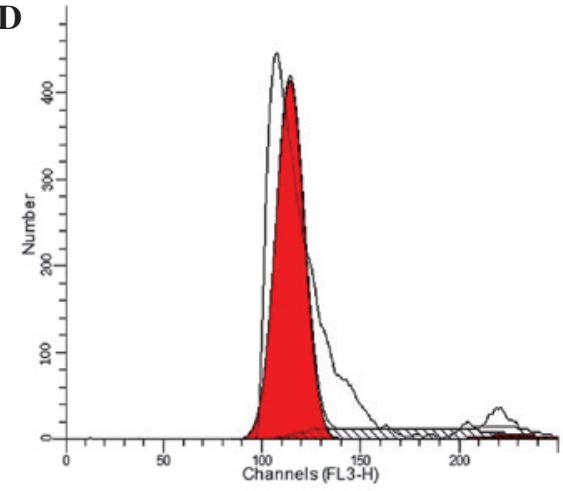

F

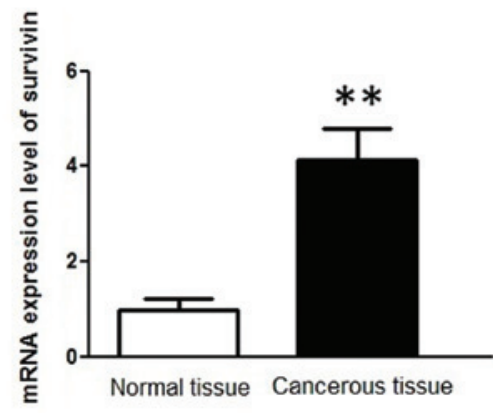

$\mathbf{H}$

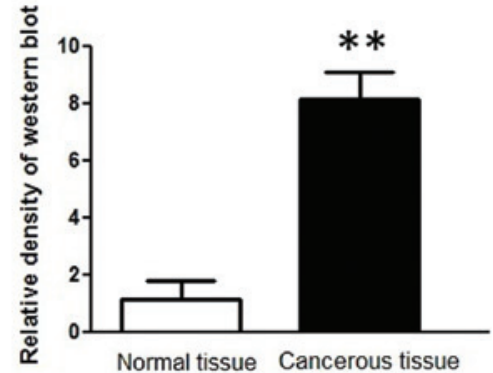

Figure 2. (A) Flow cytometric analysis of TSCC cells transfected with the controls. (B) Flow cytometric analysis of TSCC cells transfected with miR-335 mimics. (C) Flow cytometric analysis of TSCC cells transfected with miR-182 mimics. (D) Flow cytometric analysis of TSCC cells cotransfected with miR-335 and miR-182 mimics. (E) Comparison of expression patterns of miR-335 and miR-182 between the cancerous tissues and adjacent non-cancerous tissues. (F) Comparison of mRNA expression levels of survivin between the cancerous tissues and adjacent non-cancerous tissues. (G) Comparison of protein expression levels of survivin between the cancerous tissues and adjacent non-cancerous tissues, shown by representative western blotting results ( $\mathrm{n}=3$ ), (H) Comparison of protein expression levels of survivin between the cancerous tissues and adjacent non-cancerous tissues, as determined by densitometry analysis and statistical analysis. ${ }^{* *} \mathrm{P}<0.01$ vs. control. miR, microRNA; TSCC, tongue squamous cell carcinoma; mRNA, messenger RNA.

described, miR-335 is down-regulated in several types of tumors (29-32), indicating complicated physiopathological roles of this miRNA during tumorigenesis. In particular, it has been shown that miR-335 targets retinoblastoma 1 and regulates cell proliferation in a p53-dependent manner by inducing apoptosis or cell cycle arrest (33). miR-335 has previously been reported to suppress metastasis of human breast cancer cells through targeting of SRY-box 4, without affecting its proliferative ability (34). Shu et al reported that miR-335 functioned as a tumor promoter in conferring tumorigenic features, such as growth and invasion on malignant astrocytoma, and additional investigation revealed that 
miR-335 targets disheveled-associated activator of morphogenesis 1 at the post-transcriptional level (17).

Human miR-182 is mapped to chromosome $7 \mathrm{q} 32$ and is transcribed from the cluster of the miR-183 family that has been extensively researched in a variety of human cancers $(35,36)$. In certain studies, miR-182 was reported to act as an oncogene in several types of human cancers; by contrast, miR-182 demonstrated tumor suppressive activity in human gastric adenocarcinoma, lung adenocarcinomas and posterior uveal melanoma (37-39). miRNAs have been shown to frequently regulate numerous target genes with possible counteracting functions (40). Thus, the documented conflicting roles of miR-335 and miR-182 in the development and progression of malignancies may be attributed to the cell context-dependent balance among the network of directly regulated genes and the cancer type-specific activated or inactivated signaling pathways. Even though the function of miR-335 and miR-182 has been extensively studied in numerous cancer types, the roles of these miRNAs in the development of TSCC remain mostly unknown.

In the present study increased miR-335 or miR-182 expression was identified to significantly suppress TSCC cell proliferation in a TSCC cell line. The results of flow cytometry analysis demonstrated that the growth of TSCC cells transfected with the miR-335 or miR-182 mimic was arrested at the $\mathrm{G} 1$ phase with a corresponding reduction in the percentage of cells in the $\mathrm{S}$ phase (Fig. 1). By searching the miRNA databases miRanda and TargetScan for the candidate genes of the two miRs and functionally analyzing and screening the results, the present study identified survivin as a mediator of the inhibitory effect of the two miRs on the cell behavior.

Three basic roles have been identified for survivin in the regulation of cell behavior, consisting of control of cell division, promotion of cell cycle and inhibition of apoptosis (41). Survivin, a member of the IAP family, is well-known for its inhibitory role in the regulation of apoptosis and the cell cycle (7-9). Studies have demonstrated that elevated expression of survivin promoted the development and progression of malignancy (42). It is generally agreed that the cell cycle is under the control of a number of dynamically expressed genes. Survivin is expressed at high levels during the $\mathrm{G} 2 / \mathrm{M}$ phase and has been shown to play an important role in cell cycle progression (43). Survivin can accelerate the $\mathrm{S}$ phase by interacting with cyclin-dependent kinase 4, a cell cycle regulator (44). Accordingly, downregulation of wild-type survivin using RNA interference evidently caused cell cycle arrested in the G1/S phase (45). It has been shown that the expression of survivin is an early event during malignant transformation and conversion of the oral mucosa in rats exposed to carcinogens (45). A well-studied G to C polymorphism (rs9904341) in the promoter region of the survivin gene may increase the expression of the mRNA and protein of survivin by disrupting the binding site of the cell cycle-dependent element/cell cycle gene homology region repressor, and this polymorphism is associated with an increased risk of various malignancies $(46,47)$.

The expression patterns of miR-335, miR-182 and survivin were also examined and compared between the cancerous tissues and adjacent non-cancerous tissues, showing that miR-335 and miR-182 expression were significantly underexpressed and survivin expression was significantly upregulated in the TSCC cell line. The mechanism of underexpression of the two miRs in TSCC was investigated and the present findings revealed that genetic copy number loss at the locus of the two miRs was one mechanism by which miR-335 expression is silenced in metastatic cells. In addition, epigenetic modification also plays a role in the silencing of the two miRs.

In conclusion, the present study revealed a negative regulatory role of miR-335 and miR-182 in the proliferation of TSCC cells via targeting of survivin. Therefore, miR-335 and miR-182 may be novel therapeutic tools that may assist in the treatment of TSCC.

\section{Acknowledgements}

The present study was fully sponsored by the Natural Science Foundation of China (grant no. 81372844).

\section{References}

1. Jemal A, Siegel R, Ward E, Murray T, Xu J and Thun MJ: Cancer statistics, 2007. CA Cancer J Clin 57: 43-66, 2007.

2. Yuen PW, Lam KY, Chan AC, Wei WI and Lam LK: Clinicopathological analysis of local spread of carcinoma of the tongue. Am J Surg 175: 242-244, 1998.

3. Po Wing Yuen A, Lam KY, Lam LK, Ho CM, Wong A, Chow TL, Yuen WF and Wei WI: Prognostic factors of clinically stage I and II oral tongue carcinoma-A comparative study of stage, thickness, shape, growth pattern, invasive front malignancy grading, Martinez-Gimeno score and pathologic features. Head Neck 24: 513-520, 2002.

4. Bartel DP: MicroRNAs: Genomics, biogenesis, mechanism and function. Cell 116: 281-297, 2004.

5. Thai TH, Calado DP, Casola S, Ansel KM, Xiao C, Xue Y, Murphy A, Frendewey D, Valenzuela D, Kutok JL, et al: Regulation of the germinal center response by microRNA- 155 . Science 316: 604-608, 2007.

6. Brennecke J, Hipfner DR, Stark A, Russell RB and Cohen SM: Bantam encodes a developmentally regulated microRNA that controls cell proliferation and regulates the proapoptotic gene hid in Drosophila. Cell 113: 25-36, 2003.

7. Poy MN, Eliasson L, Krutzfeldt J, Kuwajima S, Ma X, Macdonald PE, Pfeffer S, Tuschl T, Rajewsky N, Rorsman P and Stoffel M: A pancreatic islet-specific microRNA regulates insulin secretion. Nature 432: 226-230, 2004.

8. Holland H, Koschny T, Ahnert P, Meixensberger J and Koschny R: WHO grade-specific comparative genomic hybridization pattern of astrocytoma-a meta-analysis. Pathol Res Pract 206: 663-668, 2010.

9. Segura MF, Hanniford D, Menendez S, Reavie L, Zou X, Alvarez-Diaz S, Zakrzewski J, Blochin E, Rose A, Bogunovic D, et al: Aberrant miR-182 expression promotes melanoma metastasis by repressing $\mathrm{FOXO} 3$ and microphthalmia-associated transcription factor. Proc Natl Acad Sci USA 106: 1814-1819, 2009.

10. Lowery AJ, Miller N, Dwyer RM and Kerin MJ: Dysregulated miR-183 inhibits migration in breast cancer cells. BMC Cancer 10: 502, 2010.

11. Yu S, Lu Z, Liu C, Meng Y, Ma Y, Zhao W, Liu J, Yu J and Chen J: miRNA-96 suppresses KRAS and functions as a tumor suppressor gene in pancreatic cancer. Cancer Res 70: 6015-6025, 2010.

12. Lu B, Mu Y, Cao C, Zeng F, Schneider S, Tan J, Price J, Chen J, Freeman M and Hallahan DE: Survivin as a therapeutic target for radiation sensitization in lung cancer. Cancer Res 64: 2840-2845, 2004.

13. Kawasaki H, Toyoda M, Shinohara H, Okuda J, Watanabe I, Yamamoto T, Tanaka K, Tenjo T and Tanigawa N: Expression of survivin correlates with apoptosis, proliferation and angiogenesis during human colorectal tumorigenesis. Cancer 91: 2026-2032, 2001.

14. Fan LF, Dong WG, Jiang CQ, Qian Q and Yu QF: Role of Hypoxia-inducible factor-1 alpha and Survivin in colorectal carcinoma progression. Int J Colorectal Dis 23: 1057-1064, 2008. 
15. Nassar A, Sexton D, Cotsonis G and Cohen C: Survivin expression in breast carcinoma: Correlation with apoptosis and prognosis. Appl Immunohistochem Mol Morphol 16: 221-226, 2008.

16. Liu L, Zhang M and Zou P: Expression of PLK1 and survivin in diffuse large B-cell lymphoma. Leuk Lymphoma 48: 2179-2183, 2007.

17. Shu M, Zheng X, Wu S, Lu H, Leng T, Zhu W, Zhou Y, Ou Y, Lin X, Lin Y, et al: Targeting oncogenic miR-335 inhibits growth and invasion of malignant astrocytoma cells. Mol Cancer 10: 59, 2011.

18. Zhang S, Kim K, Jin UH, Pfent C, Cao H, Amendt B, Liu X, Wilson-Robles $\mathrm{H}$ and Safe S: Aryl hydrocarbon receptor agonists induce microRNA-335 expression and inhibit lung metastasis of estrogen receptor negative breast cancer cells. Mol Cancer Ther 11: 108-118, 2012.

19. Xu Y, Zhao F, Wang Z, Song Y, Luo Y, Zhang X, Jiang L, Sun Z, Miao Z and Xu H: MicroRNA-335 acts as a metastasis suppressor in gastric cancer by targeting Bcl-w and specificity protein 1 . Oncogene 31: 1398-1407, 2012.

20. Shen Z, Zhan G, Ye D, Ren Y, Cheng L, Wu Z and Guo J: MicroRNA-34a affects the occurrence of laryngeal squamous cell carcinoma by targeting the antiapoptotic gene survivin. Med Oncol 29: 2473-2480, 2012.

21. Livak KJ and Schmittgen TD: Analysis of relative gene expression data using real-time quantitative PCR and the $2^{-\Delta \Delta C T}$ method. Methods 25: 402-408, 2001.

22. John B, Enright AJ, Aravin A, Tuschl T, Sander C and Marks DS Human MicroRNA targets. PLoS Biol 2: e363, 2004.

23. Lewis BP, Burge CB and Bartel DP: Conserved seed pairing, often flanked by adenosines, indicates that thousands of human genes are microRNA targets. Cell 120: 15-20, 2005.

24. Ventura A and Jacks T: MicroRNAs and cancer: Short RNAs go a long way. Cell 136: 586-591, 2009.

25. Liu ZF, Liang ZQ, Li L, Zhou YB, Wang ZB, Gu WF, Tu LY and Zhao J: MiR-335 functions as a tumor suppressor and regulates survivin expression in osteosarcoma. Eur Rev Med Pharmacol Sci 20: 1251-1257, 2016.

26. Lu Y, Yang H, Yuan L, Liu G, Zhang C, Hong M,Liu Y,Zhou M, Chen $\mathrm{F}$ and $\mathrm{Li} \mathrm{X}$ : Overexpression of miR-335 confers cell proliferation and tumour growth to colorectal carcinoma cells. Mol Cell Biochem 412 $235-245,2016$

27. Sun J, Ji J, Huo G, Song Q and Zhang X: miR-182 induces cervical cancer cell apoptosis through inhibiting the expression of DNMT3a. Int J Clin Exp Pathol 8: 4755-4763, 2015.

28. Wallis CJ, Gordanpour A, Bendavid JS, Sugar L, Nam RK and Seth A: MiR-182 Is associated with growth, migration and invasion in prostate cancer via suppression of FOXO1. J Cancer 6: 1295-1305, 2015.

29. Marsh EE, Lin Z, Yin P, Milad M, Chakravarti D and Bulun SE: Differential expression of microRNA species in human uterine leiomyoma versus normal myometrium. Fertil Steril 89: 1771-1776, 2008.

30. Ronchetti D, Lionetti M, Mosca L, Agnelli L, Andronache A, Fabris S, Deliliers GL and Neri A: An integrative genomic approach reveals coordinated expression of intronic miR-335, miR-342 and miR-561 with deregulated host genes in multiple myeloma. BMC Med Genomics 1: 37, 2008.

31. Uziel T, Karginov FV, Xie S, Parker JS, Wang YD, Gajjar A, He L, Ellison D, Gilbertson RJ, Hannon G and Roussel MF: The miR-17 92 cluster collaborates with the Sonic Hedgehog pathway in medulloblastoma. Proc Natl Acad Sci USA 106: 2812-2817, 2009.
32. Wang YX, Zhang XY, Zhang BF, Yang CQ, Chen XM and Gao HJ: Initial study of microRNA expression profiles of colonic cancer without lymph node metastasis. J Dig Dis 11: 50-54, 2010

33. Scarola M, Schoeftner S, Schneider C and Benetti R: miR-335 directly targets $\mathrm{Rb} 1(\mathrm{pRb} / \mathrm{p} 105)$ in a proximal connection to p53-dependent stress response. Cancer Res 70: 6925-6933, 2010.

34. Tavazoie SF, Alarcón C, Oskarsson T, Padua D, Wang Q, Bos PD, Gerald WL and Massagué J: Endogenous human microRNAs that suppress breast cancer metastasis. Nature 451: $147-152,2008$

35. Zhu W, Zhou K, Zha Y, Chen D, He J, Ma H, Liu X, Le H and Zhang Y: Diagnostic Value of Serum miR-182, miR-183, miR-210, and miR-126 Levels in Patients with Early-Stage Non-Small Cell Lung Cancer. PLoS One 11: e0153046, 2016.

36. Jin ZB, Hirokawa G, Gui L, Takahashi R, Osakada F, Hiura Y, Takahashi M, Yasuhara $\mathrm{O}$ and Iwai N: Targeted deletion of miR-182, an abundant retinal microRNA. Mol Vis 15: 523-533, 2009.

37. Yan D, Dong XD, Chen X, Yao S, Wang L, Wang J, Wang C, Hu DN, Qu J and Tu L: Role of microRNA-182 in posterior uveal melanoma: Regulation of tumor development through MITF, BCL2 and cyclin D2. PLoS One 7: e40967, 2012.

38. Sun Y, Fang R, Li C, Li L, Li F, Ye X and Chen H: Hsa-mir-182 suppresses lung tumorigenesis through down regulation of RGS17 expression in vitro. Biochem Biophys Res Commun 396: 501-507, 2010

39. Kong WQ, Bai R, Liu T, Cai CL, Liu M, Li X and Tang H: MicroRNA-182 targets cAMP-responsive element-binding protein 1 and suppresses cell growth in human gastric adenocarcinoma. FEBS J 279: 1252-1260, 2012.

40. Yi R, Li Y, Wang FL, Miao G, Qi RM and Zhao YY: MicroRNAs as diagnostic and prognostic biomarkers in colorectal cancer. World J Gastrointest Oncol 8: 330-340, 2016

41. Li F and Brattain MG: Role of the survivin gene in pathophysiology. Am J Pathol 169: 1-11, 2006.

42. Altieri DC: Survivin, cancer networks and pathway-directed drug discovery. Nat Rev Cancer 8: 61-70, 2008.

43. Li F and Altieri DC: The cancer antiapoptosis mouse survivin gene: Characterization of locus and transcriptional requirements of basal and cell cycle-dependent expression. Cancer Res 59: 3143-3151, 1999.

44. Ito T, Shiraki K, Sugimoto K, Yamanaka T, Fujikawa K, Ito M, Takase K, Moriyama M, Kawano H, Hayashida M, et al: Survivin promotes cell proliferation in human hepatocellular carcinoma. Hepatology 31: 1080-1085, 2000.

45. Zhang R, Wang T, Li KN, Qin WW, Chen R, Wang K, Jia LT, Zhao J, Wen WH, Meng YL, et al: A survivin double point mutant has potent inhibitory effect on the growth of hepatocellular cancer cells. Cancer Biol Ther 7: 547-554, 2008.

46. Xu Y, Fang F, Ludewig G, Jones G and Jones D: A mutation found in the promoter region of the human survivin gene is correlated to overexpression of survivin in cancer cells. DNA Cell Biol 23: 527-537, 2004.

47. Jang JS, Kim KM, Kang KH, Choi JE, Lee WK, Kim CH, Kang YM, Kam S, Kim IS, Jun JE, et al: Polymorphisms in the survivin gene and the risk of lung cancer. Lung Cancer 60: 31-39, 2008. 